\title{
Doing Qualitative Studies, Using Statistical Reasoning
}

\author{
Kristensen, Tore; Gabrielsen, Gorm
}

Document Version

Final published version

Published in:

Proceedings of DRS 2016 International Conference

Publication date:

2016

License

Unspecified

Citation for published version (APA):

Kristensen, T., \& Gabrielsen, G. (2016). Doing Qualitative Studies, Using Statistical Reasoning. In P. Lloyd, \& E. Bohemia (Eds.), Proceedings of DRS 2016 International Conference: Design + Research + Society - FutureFocused Thinking (Vol. 3, pp. 1211-1222). Design Research Society. Proceedings of DRS International Conference

Link to publication in CBS Research Portal

\section{General rights}

Copyright and moral rights for the publications made accessible in the public portal are retained by the authors and/or other copyright owners and it is a condition of accessing publications that users recognise and abide by the legal requirements associated with these rights.

Take down policy

If you believe that this document breaches copyright please contact us (research.lib@cbs.dk) providing details, and we will remove access to the work immediately and investigate your claim. 


\title{
Future-Focused Thinking
}

\section{Doing qualitative studies, using statistical reasoning}

\author{
First name Last name ${ }^{*}$ TORE KRISTENSEN AND GORM GABRIELSEN \\ ${ }^{a}$ Affiliation name BOTH \\ ${ }^{b}$ Affiliation name COPENHAGEN BUSINESS SCHOOL \\ *Corresponding author e-mail: TK.MARKTG@CBS.DK
}

\begin{abstract}
Qualitative studies are associated with interviews, focus groups and observations. We introduce experiments as a way of dealing with such studies. In contrast to the common focus on how many respondents choose a particular behaviour we focus on how much a design affect the individual. This is often concerned with analysing the effect of a design. The approach is bottom up, in that the inferences are concerned with each individual. This enables us to look at the variation between people. We consider the common preference profile, defined as that part of the individual preference profiles which is shared by all individuals. A variation seen by the individual means that the message is received with its complexity and meaningfulness, while a big ideosyncratic variations means people understand different things and a Babylonian confusion is the outcome. Findings may be generalized after the effect has been measured at an individual level.
\end{abstract}

Keywords: qualitative data, variation, idiosyncratic, experiment

\section{Introduction}

Among the last discussion we had with John Heskett was the methodology of design research. Heskett contributed with many insights in design using both theoretical and empirical methods. John was indeed a competent theoretician, and still he was more an Aristotelian than a Platonist. This means he preferred theories developed via empirical research and often close to practical ways of doing design.

Designing is very often itself a form of experimentation by which we means concepts are developed and via trial and error one find how well a particular design does in a practical setting. Obviously many research methods are also used. In some cases design are investigated by marketing research with those standards and sometimes with the growing standards of design research. These methods may in general be labelled qualitative and among the preferred are focus groups, ethnographic observations, various interview techniques etc. 
In this paper we suggest a methodology developed in sensorics, often associated with food research and aiming at experiments to judge whether a particular taste, color, feeling of substance is working well. John saw clearly the advantage of using such methods for design research. However, many experimental methods are characterized by the used of statistics and very often the focus on aggregate data such as comparing a core group with an alternative group to see if the averages in the two groups differed significantly from each other. The challenge with this is that while averages may be meaningful if you consider how rich people are and how much they are willing to spend for a particular issue, the likings or preferences cannot be seen as averages. It makes no meaning to say the average preference for a chair is such and such. We accept that often the expression is that such and such a fraction of a population prefer one design to another. But also in these case, we have a suspicion that the full story is not told. People have individual preferences. This is an obvious reason for using qualitative methods like interviews.

However, could there be a way to solve this, to use statistical methods as they are often seen as more reliable or even valid than qualitative interviews by people outside the sphere of design?

We believe that is so and we will, in John Heskett's spirit explore how we may use such statistical methods for qualitative studies, emphasizing that people do not have average preferences for design; they are individuals.

Next section contains some conceptual explanations of how we regard designs from a cognitive viewpoint and stage designs for further explorations.

\section{Our approach}

Most studies in marketing operate at a market level, which also becomes frequently the condition for design work. This means that the aggregate or weighted average consumer is the focus. Having already made this aggregation as the "top-down approach" indicates, it is not feasible to take the individuals apart and explore how they differ. This is so because already the concepts and the methodology is founded at the aggregate level. The differences have been eliminated and replaced by an assumed normal distribution. We depart from that and start our investigations at an individual level. This means, in the "bottom up" approach we keep the individual variation or differences intact for further analysis. We base our concept and methodology at the individual level. Only then, understanding what happens for the single individual we can eventually aggregate to see the consequences at a market level. We explore a procedure that enables the marketer to estimate the effect of a marketing message like a mission statement in a logo at the level of a single individual. This is a prior to market test, with its own realism. The procedure can easily be extended to products, where both quality and price (willingness to pay) are issues and it can also be used as a follow-up after the round of pre-test. 
We want to explore the implications of consumers being different; and we want to quantify differentness or simply individuality. We want to investigate about more or less differences between people in a market.

\section{Statistical reasoning}

A common statistical reasoning in a market context is concerned with a top-down approach, where the analysis ex ante has stated a condition that certain criteria are likely to characterize the sample or population. The reasoning is then concerned with the number of people e.g. belonging to certain categories or segments. If the challenge concerns aggregate data or analysis at such a level, this may be satisfactory. On the other hand, a designer often aims at considering individual needs. If this is the case, the average may be an insufficient approximation, because it means dropping important information from the investigationinformation that could mean a more flexible and varied design. It may mean additional challenges, but it is better to face these challenges in the design process than learn that potential customers dropped the product altogether. To satisfy individual users, real individuals should be considered. And statistical methods and reasoning is useful in keeping the trace of the individual.

Method individualism is argued by e.g. Elster (2000) as a foundation for social theory. Everything should be explained at an individual level. Also Ziliak and McCloskey (2008) share this view, indicating that relying on aggregate phenomena may create inferential constructions. That is explanations that may be statistically significant, but does not refer to real events or individuals. Concepts like median voters, average consumers may be examples of constructions that may not be identified in a real setting. This means choices should be explained at an individual level. This means from the outset that we are not measuring how many people belong to a certain segment, but how much they value a particular quality in a choice situation. This is central when considering demand. This issue will be considered when we introduce examples of statistical reasoning.

The presentation will introduce a set of examples concerned with studying the variation in various markets and their significance. The contrast will show how much is lost by assuming that an average user will be sufficient to cover the full variation. In particular, the so-called "long tail", which is an asymmetric distribution, will show that some users are very far from the average and the average solution for them would be highly unsatisfactorily.

\section{Cognitive models may explain spontaneous order}

Designs are perceived and managed in the mind of the prospective customer. This means that the perception takes place at an individual level. We assume a problem-solving and active perceiver rather than a passive response. Rather than to test every aesthetic element independently, everything is seen as a whole and measured according to an idiosyncratic (or random) processing. In the case of a logo, the situation concerned is not so much the actual product as the producer or marketer who wants to signal a brand or just availability. The 
user or customer uses a logo to inform themselves about possible offers and to make a choice (Henderson and Cote 1998). This is complementary to the marketer who informs by the intentions of the visual elements, often expressed as a mission statement. For the user or customer, the availability of the logo is an affordance, enabling them to make a choice and subsequently experience the benefit from a choice of an artefact, object, service or experience.

Since a market transmits information, it may also become a cognitive mechanism (Clark 1996) and people may use the information to create knowledge According to him Johnson (1987), pervasive features of bodily experience of the environments comes from the perception of the human body and forces dealing with movement, keeping a position, using the body for practical purposes. This Johnsons (1987) refers to as containment and boundedness in bodily experiences and writes,

"Our encounter with containment and boundedness is one of the most pervasive features of our bodily experience. We are intimately aware of our bodies as threedimensional containers into which we put certain things (food, water, air) and out of which other things emerge (food and water wastes, air, blood, etc.). From the beginning, we experience constant physical containment in our surroundings (those things that envelop us). We move in and out of rooms, clothes, vehicles, and numerous kinds of bounded spaces. We manipulate objects, placing them in containers (cups, boxes, cans, bags, etc.). In each of these cases there are repeatable spatial and temporal organizations. In other words, there are typical schemata for physical containment." (p. 21).

The proper term for this is image schemata, describing the repeated and often stereotypical action (p. 65). Several image schemata are more complex than this indicates, and it also makes sense that human beings need to name them to create meaning for themselves and to share with others. With image-schemata as the elementary building bloc, metaphors and metonyms provides the naming (Lakoff 1987, Lakoff and Johnson 1999). Terms like force, move, transport, fall, fly, take off all rely on image schemata and using metaphors we are able to refer to specific instantiations. Also formal or propositional models serve this function by setting standards for measurement and human scales of everything. Both outside and inside markets, humans may communicate using tacitly agreed standards like an inch, a foot, intimate distances, dancing, references to other language areas (likings) and focus of particular issues (the Hill or Number ten) of complex objects as well as being there, doing bodily things. As Woody Allen has stressed, " $80 \%$ of success is just being there".

What constitutes a good experience? When is a design good for the person who has the experience? A process view is proposed to indicate that a good design is "easy on the mind", Mayvis and Jervis (2001) who adds a mediating variable, "fluency" meaning a logo perceived with a minimum of cognitive work. Cognitive work is typically measured by response times, how fast or easily respondents can get the message (Reber, Winkielman and Schwarz (2004).They claim that what is easy on the mind may be preferred or simply found more 
beautiful that alternatives requiring more struggle. The concept has also been used in connection with branding (Lee and Labroo 2006). This is where logos come in. Logos promise that the company behind will deliver something valuable in the future, given that the customer accepts to buy, pay and pick up the goods. Logos are setting expectations by communicating certain visual (and verbal) key figures that refer to the product, artefact, system or experience.

With designs, we enter into a language where certain signs, symbols, shorthand, figures are used to communicate general offers and values of the company, affordances or how the people behind may enable to customer or user to take advantage of their objects, artefacts, services and experiences.

The aesthetic elements are known as gestalt forms. Henderson and Cote (1998) use these well-known principles researched during the history of aesthetics by Gestalt theorists such as Gombrich (1972) and Arnheim (1974). They were simple principles shown to attract attention and to create beauty. Gestalt phenomena are considered to be of a biological and even universal validity (Ramachandran and Hirstein 1999).

\section{The designers' perspective}

The design seen from the perspective of the designer or company using design?

With Searle (1969) we may say that to design and communicate a logo is a locutionary act that is the basic act of producing a performative (Searle 1989) or meaningful utterance e.g. a verbal or visual statement within a community. Such an utterance sets an expectation or hopes for the future. A design has a purpose, referred to as an illocutionary act, which means to express a statement that promises (or primes) a message (future satisfaction) to a receiver, hopefully a prospective customer.

Finally, perlocutionary act is the effect of the utterance, what the customer hears and perceives. How does the individual perceive the meaning of the logo? For an individual a rich content may mean that the perlocutionary content is close to the illocutionary; or the interpretation is close to the intent.

Meanings may be embodied, that is experienced and often with limited conscious deliberation. Zajonc (2003) have explained that in order to make a judgment, respondents need not be conscious deliberation about the perception. A reason for the many logos most people actually know is that they have been exposed to the many times in their lives and probably accepted the quality of the product or brand.

\section{Treating the user or consumer}

Most market communication, including logos (Henderson and Cote 1998, Veryzer and Hutchinson 1998, Hynes 2009) treat the prospective customer as a respondent. We take a different approach looking at the active problem solving customer who looks for affordances (Gibson 1978) and enablers in the design. 
A choice depends on deliberations and personal negotiations of values, such as exchanges of gains and losses, the explanations and story of why the choice of design is based on perception of prospective problem-solving and meaning creation (Heskett 2002). This may be the situation where a person is in a shopping situation, looking at, touching, valuing alternative goods, solutions, objects, artefacts for possible acquisition.

Our theoretical view and way of dealing with choice models is cognitive and based on embodiment and embeddedness. The first issue (e.g. Clark 2003) is based on the assumption explained above that there is a fluent transformation between choices taking place fast of slow and that conscious elements is integral to much but only as an important ingredient (Damasio 2003). A choice may sometime happen very fast (Kahnemann 2014). Embeddedness concerns typically the context in which things happens. A strong embedded network may have strong influences on how we choose as sometimes seen in emotionally strong mass-events like Superball (Chwe 2001). Here some action is strongly synchronized where people are actually present or follow the rhythm via their TV set. Other situations may rather be described as weak links (Granovetter 1973, Chwe 2001) where the network may influence people in various degrees, but rarely totally like in an emotional mass-event (Christakis and Fowler (2010).

\section{Variation between Babel and Common Knowledge}

Babel is the Christian story of how people from many cultures spoke a mutually understandable language came to work together and wanted to build a tower to "heaven". The god got angry and made it so every individual speak different languages. Confusion and lack of ability to complete the tower meant it all fell apart. The scale may encapsulate any position from which anyone acting in a regime of varying knowledge works from. The Babelian confusion is a rare situation where people are unable to communicate because nobody understands what others see or talk about. This is unrealistic for human beings since we know (Morris 1994) that body language may integrate even very remote cultures.

On the other hand recent research (Kristensen and Gabrielsen 2016) shows considerable variation in a group of people, randomly selected who were asked to consider the meaning of certain logos and their mission statements. Excluding the sampling error the idiosyncratic variation was typically concerned with $80-90$ percent so that the shared message amounted to $10-20$ percent, which means there was an overwhelming variation in the way logos were seen when respondents were shown the logos. However, when a new design was introduced in the form of an integrating language, subculture or common language, the idiosyncratic variation decreased considerably. By providing a common background for understanding, for instance a story, a particular epic, style or genre the variation was reduced considerably and more mission statements were understood.

One way of modelling this is the following scale: 

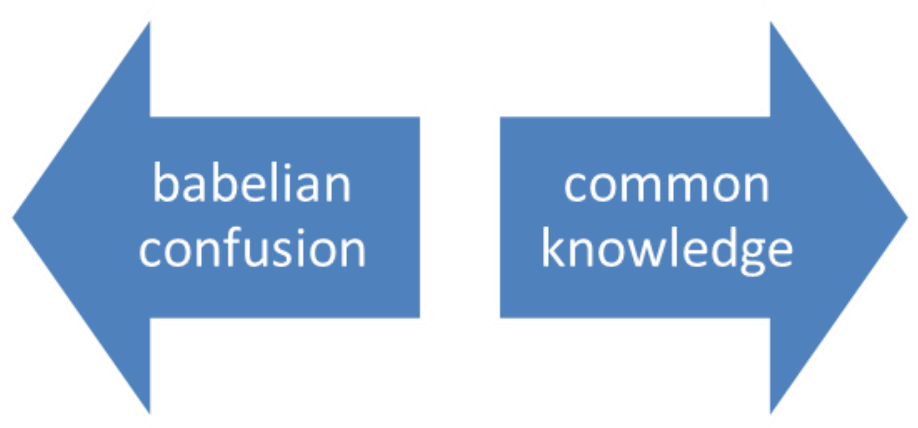

Common knowledge is the contrary situation. A vital issue is not only that people know the same (..), but that they know that the others know the same and that they know that others also know. Common knowledge is a formal concept formed within modern game theory (Lewis 1969, Aumann 1974). The extreme version may look like grand media events like Olympic Games or Super bowl, US presidential election etc. where large parts of world population are seers (Chwe 2001). Also with the birth of radio and later television, there was usually only one channel and everybody was listening or viewing the same programs. With multiple programs and channels - not even to think about the internet - that has changed dramatically and big steps have been taken in the direction of the Babelian position.

While this means less common knowledge, it does not also necessarily mean problemsexcept when it is vital for someone in particular that everybody knows everything etc. The French general De Gaulle said: How can you govern a country which has two hundred and forty-six varieties of cheese? ("Comment voulez-vous gouverner un pays qui a deux cent quarante-six variétés de fromage"? Mignon 1972). It seems common knowledge, more than Babelian confusion that supports a political system or government. Anarchists may not be happy for common knowledge (Kropotkin 1902). But there is more to it.

Also a movement towards confusion may have costs in a society in the sense that knowledge of crafts, procedures, may be less common, such as information costs, even asymmetric information (Akerlof 1980).

\section{Diversity of what?}

A major issue concerns what we may call cultural diversity. What it directly concerned depend on the analysis at hand, but such issues as values, norms and rites are examples.

A value represents a slogan capable of providing for the rationalization of action by encapsulating a positive attitude toward a purportedly beneficial state of affairs Rescher 1969 p. 9. This is indeed an intellectual or articulated way. Values are to a large degree seen as symbols or experienced (Boztepe 2007). An experienced value does normally need no articulation or explanation. For instance by travelling, tasting food from other regions, meeting people with different backgrounds provides variation in experiences which are mostly absorbed through the emotions or feelings (Damasio 2003). When experiences seem 
really odd, like culinary experiences that is taboos like in many western cultures eating horse, dogs, cats, insects or very strange do the signal come that there is something alien or potentially dangerous going on. Domestic experiences may be noted if they are particularly good or very strange.

What kind of elements creates this variation? As we suggested above, a source of variation comes from the perlocutionary utterance and another from the perlocutionary. Is one dominating the other and is there an interaction between them?

Hutchins (2004) emphasized the material anchoring of the processes, mainly referring to devises, but also behavioural design elements such as standing in a Line. In Hutchins' (2004) optic, the examples are e.g. navigation devices used by many people to actually navigate movements on the earth. But to many people the navigation is social and may include certain objects, artefacts to show they possess good taste and belong to a certain in-group (Douglas 1979, Bourdieu 1982).

\section{Introducing experiments}

We experiment with such processes as a way of exploring how prospective users or customers value the outcome when given a paired choice. "Will you take this one or that one" is based on using a computer, iPad with a screen showing visually pairs of choices with a line - scale - between. We may test a number of options and add prices, brand names and stories and use conjoint-layouts. The respondent is asked to move the cursor towards the preferred choice and move it so close to the preferred choice that the closeness indicates the strength of the preference. By letting the same respondent make paired comparisons with varying options we may estimate the respondent's rating of a number or items enabling us to rank order how strongly each object, artefact, model or prototypes is preferred by each single respondent in a sample of users. We consider such a rated rank order of objects or services a preference profile. The core is that by experimenting we can construct individual preference profiles, i.e. characterizing the single individual in contrast to characterizing a market. The data collection is web based and may be done anywhere, also when no internet is available because the connection can be made later. We need to develop a statistical program to deal automatically transforming the data to a comprehensible output with statistical tests, graphs etc. The research design needed to explain and measure how logos serve as communication elements and how effective they are, depends on a setup using the web. It is important (Louviere, Flynn and Carson 2010) that the experimental procedure reflects the real behaviour it is supposed to explore and explain. This means that a normal choice experiment, where tangible goods are to be investigated best take place in a real shopping environment and that the sequence of the experiment simulates a real behavioural process. Only if the experiment is intended to explain what happens online, should web designs be used. A common way of using picture of real objects on a pure web design is a poor research design when it is supposed to show what happens in a real shopping process. Similar should experiential studies of what the 
user or consumer experience and may feel satisfied with take place in their homes or else where such experiences take place. In the current study we use a web-based design because it is quite common that the first time a respondent meets a new logo and considers its promises take place on the web.

In a given situation to estimate the model we perform an experiment. That is we construct a setting from which we can relate the outcome of the experiment to the parameters of the model. In general there are many ways to interpret the result of an experiment so we have to be very carefully using an appropriate statistical design.

In marketing the answer to the usually posed question "How many?" relates to a population or a market; implicitly by this "counting-approach" we are measuring a market. By sampling we usually want to estimate the fraction of some properties, e.g. democrats, republicans. In this case we pretend that all democrats are "equal" and similarly that all republicans "equal". To achieve knowledge about the desired fraction from sampling no more information about the single respond is needed than whether the single respondent is a democrat or a republican. In this case the viewing ankle or the model implicitly becomes a so-called "Urn model", that is an urn with a fixed number of balls, each being either red (democrats) or green (republican). Drawing in random a number of balls we can from the sample estimate the fraction of red balls (democrats) in the population by the fraction of the observed red balls (democrats) in the sample.

The question is what is random from this viewing ankle - or this model? The balls have fixed colours (democrat or republican) and what is random is which ball is chosen.

A totally different viewing ankle is the following; we ask a person which president candidate he will vote for. In this case we can sider the question a random experiment with two possible outcomes namely democrat or republican and what is random is what the respondent answers. Again we can estimate the fraction of respondents voting for e.g. democrat by the fraction answering "democrat" on the basis of a random sample of voters. The two perspectives will in this setting lead to the "same result", as both will yield consistent estimates; however, the two perspectives are quite different from a more theoretical point of view as the consistency is based on quite different probability arguments.

The first perspective is focusing on the population, the second is focusing on the respondents in the sense that in the last case we observe respondents and from these individual observations we may - under certain conditions - aggregate to a population and giving answer to the question of fraction of democrats.

In that sense the two models are totally different - they are in the words of Kuhn incommensurable, although both view ankles can give "correct answer" to the question.

The last model is called an inferential model indicating that it is a model - whereas the Urn model is referred to as random sampling procedure (or model) - downplaying that it "of cause" is a model. 
The sampling approach cannot intuitively be extended to treat more attributes of the respondent. Doing that by giving the balls more than one color leads to an "Elaboration" procedure, being a top-down procedure initiated among others by the sociologist Lazersfeldt and others.

The inferential approach, however, can easily be extended by observing more attributes of the same respondent. In this case the result of the analysis may be aggregated to the level of the population, which is a bottom-up approach.

The basic idea is here that the sampling approach is a top-down approach whereas the model approach is a button-up.

The sampling approach is usually focused on analyzing fraction - and "explaining" why some fraction are different. It is in this model difficult to talk about variation as the variation implicitly is a function of the fractions. In this model there is no real concept of variation.

We therefore introduce continuous measures and we include several objects which are mutually compared.

In experiments we apply paired comparisons, using Thurstone (1927) scales which enables both a continuous measurement and also works at a non-numeric registration by allowing respondents to move a cursor on a screen to indicate the proportion of which $\mathrm{s} /$ he prefers one out of two options.

By variation we mean two different things. We consider the common preference profile, defined as that part of the individual preference profiles which is shared by all individuals. Further we consider how much the individual preference profile differs from the common preference profile. This difference is due to individual peculiarities and is the idiosyncratic deviance. The sum of the idiosyncratic deviances is the idiosyncratic variation, which is the variation each person attributes to a statement vs. the common variation of subjects in a market. A variation seen by the individual means that the message is received with its complexity and meaningfulness, while a big market variations means people understand different things and a Babylonian confusion is the outcome. Also differences between cultures are investigated, but found to be of limited size compared to the between subjectvariation. If the between people variation dominates, it means that there are many competing interpretations in the market and that the market is fragmented. This means the agents are limited in their ability to compare and talk about the market message.

\section{Conclusions}

The presentation will show some examples of statistical reasoning where individual needs and perception of designs differs very much from the average preference. Statistical reasoning is shown to be useful for finding these differences and to indicate how it is the qualities of the needs and their satisfaction rather than the number of people within a segment that is the essential. 


\section{References}

Ekman, Paul (1999) Basic Emotions In T. Dalgleish and M. Power (Eds.). Handbook of Cognition and Emotion. Sussex, John Wiley \& Sons, Ltd.

Elster, Jon (2007) Explaining Social Behavior More Nuts and Bolts for the Social Sciences Cambridge: Cambridge University Press

Gombrich, E.H. (1979) The Sense of Order A study in the Psychology of Decorative Art London: Phaidon

Gombrich, E.H. (1999) The Uses of Images Studies in the Social Finction of Art and Visual Communication London: Phaidon

Haramundanis, K. (1996). Why icons cannot stand alone. ACM Journal of Computer

Documentation, 20(2), 1-8.

Louviere, Jordan J., David A. Hensher and Joffre D. Swait (2000) Stated Choice Models Analysis and Applications Cambridge: Cambridge University Press

Louviere, Jordan J., Terry N. Flynn and Richard T. Carson (2010) Discreet Choice Experiments Are Not Conjoint Analysis Journal of Choice Modelling 3 (3) pp. 57 -- 72

Ziliak Stephen and Deidre McCloskey (2008) The Cult of Statistical Significance How the standard Error Costs us jobs, Justice and Lives Ann Arbor: University of Michigan Press Pittard, N., Ewing, M. \& Jevons, C. (2007). Aesthetic theory and logo design: examining consumer response to proportions across cultures. International Marketing Review, 24(4), 457-473.

Ramachandran, Vilanur and William Hirstein (1999) The Science of Beauty Journal of Consciousness Studies Vol. 6, 15 - 51

Reber, Rolf, Piotr Winkielman, and Norbert Schwarz (1998) "Effects of Perceptual Fluency on Affective Judgments," Psychological Science, 9 (1), 45-48.

Reber, Rolf, Norbert Schwarz and Piotr Winkielman (2004) Processing Fluency and Aesthetic Pleasure: Is Beauty in the eye of the Perceiver Processing Experience Social Psychology Review, Vol. 8, No. 4, pp. 364-382

Rosbergen, E., Pieters, Rik, \& Wedel, M. (1997). Visual attention to advertising: A Segment Level analysis. Journal of Consumer Research, 24(3), 305-314.

Schmitt, B.H. \& Simonson, A. (1997). Marketing aesthetics. The Free Press, New York, NY Schwartz, S. H. (1994). Are there universal aspects in the content and structure of values? Journal of Social Issues, 50, 19-45.

Searle, John (1969) Speech Acts: An Essay in the Philosophy of Language Cambridge:

Cambridge University Press

Veryzer, Robert W. and J. Wesley Hutchinson (1998) The Influence on Unity and Prototypicality On Aesthetic Responses To New Product Designs Journal of Consumer Research Vol. 24 March $374-394$

About the Authors:

Tore Kristensen is professor of strategic design at Copenhagen Business School. He has a PhD in product development he. has taught design management and strategic design since 1989. His 
research is studies in how users appreciate design and how it contributes to wealth in various ways.

Gorm Gabrielsen is a mathematician and statistician. He has research experience from forensic research, food and criminology in addition to design studies. A common approach may be found for common ways to grasp and analyse issues of how users appreciate design. 